\title{
Passive magnetic separator integrated with microfluidic mixer: Demonstration of enhanced capture efficiency
}

\author{
Lund-Olesen, Torsten; Bruus, Henrik; Hansen, Mikkel Fougt
}

Published in:

Proceedings of MEMS

Link to article, DOI:

10.1109/MEMSYS.2006.1627817

Publication date:

2006

Document Version

Publisher's PDF, also known as Version of record

Link back to DTU Orbit

Citation (APA):

Lund-Olesen, T., Bruus, H., \& Hansen, M. F. (2006). Passive magnetic separator integrated with microfluidic mixer: Demonstration of enhanced capture efficiency. In Proceedings of MEMS (pp. 386-389). IEEE. https://doi.org/10.1109/MEMSYS.2006.1627817

\section{General rights}

Copyright and moral rights for the publications made accessible in the public portal are retained by the authors and/or other copyright owners and it is a condition of accessing publications that users recognise and abide by the legal requirements associated with these rights.

- Users may download and print one copy of any publication from the public portal for the purpose of private study or research.

- You may not further distribute the material or use it for any profit-making activity or commercial gain

- You may freely distribute the URL identifying the publication in the public portal 


\title{
Passive Magnetic Separator Integrated With Microfluidic MiXer: DEMONSTRATION OF ENHANCED CAPTURE EFFICIENCY
}

\author{
T. Lund-Olesen ${ }^{*}$, H. Bruus, and M.F. Hansen \\ MIC - Department of Micro and Nanotechnology, Technical University of Denmark, \\ DTU building 345 East, DK-2800 Kongens Lyngby, DENMARK, \\ E-mail: tlo@mic.dtu.dk
}

\begin{abstract}
In this paper, we present two results: (1) a new method for quantifying the efficiency of magnetic bead separators by magnetic measurements, and (2) a new idea for designing efficient magnetic bead separators. For microfluidic magnetic separators, a limiting factor for the capture of magnetic beads is the steep decrease of the magnetic force on the beads as a function of their distance to the magnetic structures. Our idea is to integrate the magnetic separator with a microfluidic mixer to ensure that all beads are brought close to the magnetic structures. We have fabricated a magnetic separator consisting of permalloy elements adjacent to a microfluidic channel. The performance of a system with an integrated surface structured (staggered herringbone) microfluidic mixer is compared to that of an equivalent system without the mixer. It is quantitatively demonstrated that the mixer significantly enhances the bead capture-andrelease efficiency.
\end{abstract}

\section{INTRODUCTION}

Microsystems containing magnetic structures for the separation of magnetic microbeads coated with biomolecules are receiving growing interest for bioanalysis [1-7]. However, the magnetic force on beads decays rapidly with the distance from magnetic structures; as a consequence mainly beads close to these structures will be captured thus lowering the capture efficiency of the devices. We present and demonstrate a novel microsystem design that solves this problem: A surface structured (staggered herringbone) microfluidic mixer [8] is integrated with a passive magnetic separator. By integrating magnetic separators with microfluidic mixers, two advantages are obtained: (1) All magnetic beads are brought close to the capturing sites, thus increasing capture efficiency. (2) The mixer creates a transverse fluid flow, which facilitates bead release by forcing the beads away from the slowly moving fluid at the sidewalls. We have verified this experimentally by comparing quantitative measurements of the capture-and release efficiency for systems with and without mixer structures.

In the literature, the capture of magnetic beads has been reported in several ways: visual inspection of captured and escaping beads [2, 3], fluorescence microscopy [4] measured inductance changes in electromagnets [5], simulations [6] and estimates of relative amounts of non-magnetic and magnetic beads before and after magnetic release of a mixed bead sample [7]. Common for these reports is that they do not consider the beads that stick to the magnetic structures and/or settle in the system and fluidic setup. We have performed measurements of the absolute amounts of beads using a vibrating sample magnetometer (VSM). By collecting and comparing the absolute amounts of beads entering the system and released from the system it is possible to obtain the capture-and-release efficiencies and assess sample loss in the fluidic setup.

\section{SYSTEM DESIGN}

Figure 1 shows a micrograph of a system and the two insets indicate the mixer principle. On each side of the microfluidic channel $\left(l \times w \times h=13500 \times 400 \times 80 \mu^{3}\right)$ there are 30 permalloy magnetic elements $\left(l \times w \times h=4300 \times 150 \times 50 \mu \mathrm{m}^{3}\right)$ with a distance to the channel of $20 \mu \mathrm{m}$. The herringbone mixer structures are fabricated as ridges at the bottom of the channel. Each herringbone consists of a wide and a narrow part angled in a vertex of $\pm 45^{\circ}$. The herringbone ridges are $30 \mu \mathrm{m}$ high, $21 \mu \mathrm{m}$ wide, and are spaced $60 \mu \mathrm{m}$ apart in groups of five. The position of the vertex alternates between left and right from one group to the next.

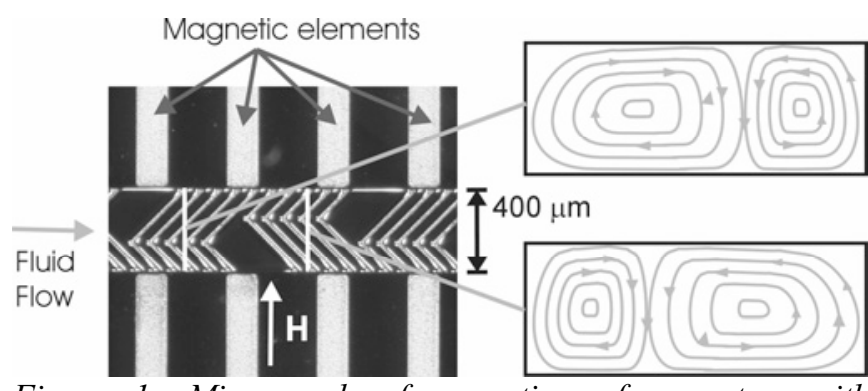

Figure 1: Micrograph of a section of a system with integrated mixer structures. The directions of the external magnetic field and the fluid flow are indicated. On the right are shown schematics of the transverse fluid flow at two cross sections in the channel.

\section{SYSTEM FABRICATION}

The fabrication of the systems is performed in silicon using a technique pioneered by $W u$ et al. [9] and further developed by Smistrup et al. [4]. The magnetic elements are electroplated using etched structures in the silicon as electroplating mold. The fabrication of the system with the integrated mixer structures is outlined in Figure 2. The system without mixer was fabricated simultaneously by omitting the thick photoresist masking for the herringbones in the fluidic channel seen in Figure 2C. 


\section{EXPERIMENTAL}

\section{Bead capture experiments}

Bead capture experiments were performed using a $2 \times 10^{7}$ beads/mL solution of $1 \mu \mathrm{m}$ MyOne beads [10]. A homogeneous magnetic field of $50 \mathrm{mT}$ was provided by a table-top electromagnet. To carry out an experiment, the electromagnet was switched on and a well-defined bead solution volume of $110 \mu \mathrm{L}$ was driven into the system using a buffer flow of $20 \mu \mathrm{L} / \mathrm{min}$ from a syringe pump. The fluidic setup is described in [11]. The buffer flow was maintained until no more beads entered the system. To release the beads, the buffer flow rate was increased to $50 \mu \mathrm{L} / \mathrm{min}$ and the field from the electromagnet was reduced to zero. Subsequently, the buffer with the released beads was collected in thinwalled plastic tubes. Micrographs were taken before, under and after the bead capture and release experiments using a Leica MZ FL III stereomicroscope equipped with a Sony DFW-X710 CCD camera.
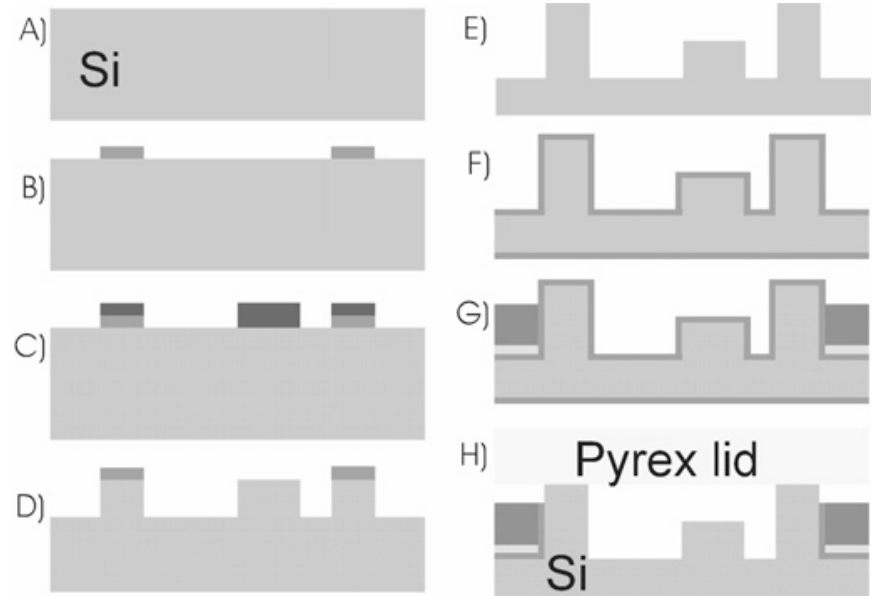

Figure 2: Outline of the fabrication sequence. A) The starting $\mathrm{Si}$ wafer. B) $\mathrm{A} \mathrm{SiO}$ etch mask is fabricated. C) A thick photo resist etch mask is applied on top of the oxide mask. D) A deep reactive ion etch (DRIE) is performed and the resist is stripped. E) A second DRIE and an oxide etch are performed. F) An oxide is grown for electrical insulation. $G)$ A metal seed layer is selectively deposited in the element trenches and permalloy is grown by electroplating. H) The oxide is etched, and a pyrex wafer is anodically bonded to the system.

\section{Bead quantification using magnetic measurements}

To characterize the system, a new method was devised for quantifying the amount of beads in samples collected in thinwalled plastic tubes. This was done by magnetic measurements in a LakeShore 7407 vibrating sample magnetometer. Such measurements are not trivial as typical amount of beads collected in the experiments have a saturation magnetic moment of $m \sim 30 \mathrm{nAm}^{2}$, which is a small value. The equipment therefore has to be sensitive and more sig- nificantly, background contributions and magnetic impurities need to be controlled. Ferromagnetic dust particles can easily have a magnetic moment of 3-4 $\mathrm{nAm}^{2}$ and the sample handling is therefore critical.

We established the following procedure for quantifying amounts of magnetic beads: Starting with a bead solution in the plastic tube, the beads are collected at the bottom of the tube by placing it on top of a $\mathrm{NdFeB}$ magnet. The supernatant is then removed and the beads are dried and fixed at the bottom of the tube using low-viscosity glue.

The measurements of magnetic moment in the VSM were sensitive to the sample position. Due to the low signal from the samples, the sample positioning was carried out with a reference plastic tube with a larger amount of beads. Using a custom made sample holder, care was taken to place other samples with the bead spot at the same position as that of the reference sample. All measurements were corrected for the background contributions from the sample holder and dia-/paramagnetic contributions from the plastic tube and the glue. Control measurements on empty plastic tubes were carried out frequently to ensure that the sample mount was not contaminated.

To validate the method and estimate its reliability, control experiments were carried out repeatedly on empty plastic tubes, and tubes with different well-defined volumes of the bead solution.

\section{Quantification of bead capture-and-release efficiency}

To measure the bead capture-and-release efficiencies and to evaluate whether the full amount of beads entered the microfluidic system, the following experiments were carried out - all with an initial bead solution volume of $110 \mu \mathrm{L}$ and a buffer flow rate of $20 \mu \mathrm{L} / \mathrm{min}$ :

(1) The beads were collected at the input fluidic connection of the chip holder.

(2) The beads were collected at the output fluidic connection of the chip holder without applying a magnetic field.

(3) The beads were collected at the output fluidic connection of the chip holder after magnetic capture in the microfluidic system.

Subsequently, the amounts of collected beads in the various experiments were quantified using the described magnetic measurements and yielded the saturation magnetic moments $m_{(1)}, m_{(2)}$ and $m_{(3)}$. Ideally, the two first experiments should give $100 \%$ compared to the introduced number of beads, while the third experiment yields the capture efficiency. In a less ideal situation, e.g., if beads get stuck in the fluidic setup and never enter the system, the capture-and-release efficiency is defined as $m_{(3)} / m_{(1)}$ and if some are also lost at the outlet in the system, the capture-and-release efficiency should be defined as $m_{(3)} / m_{(2)}$. 


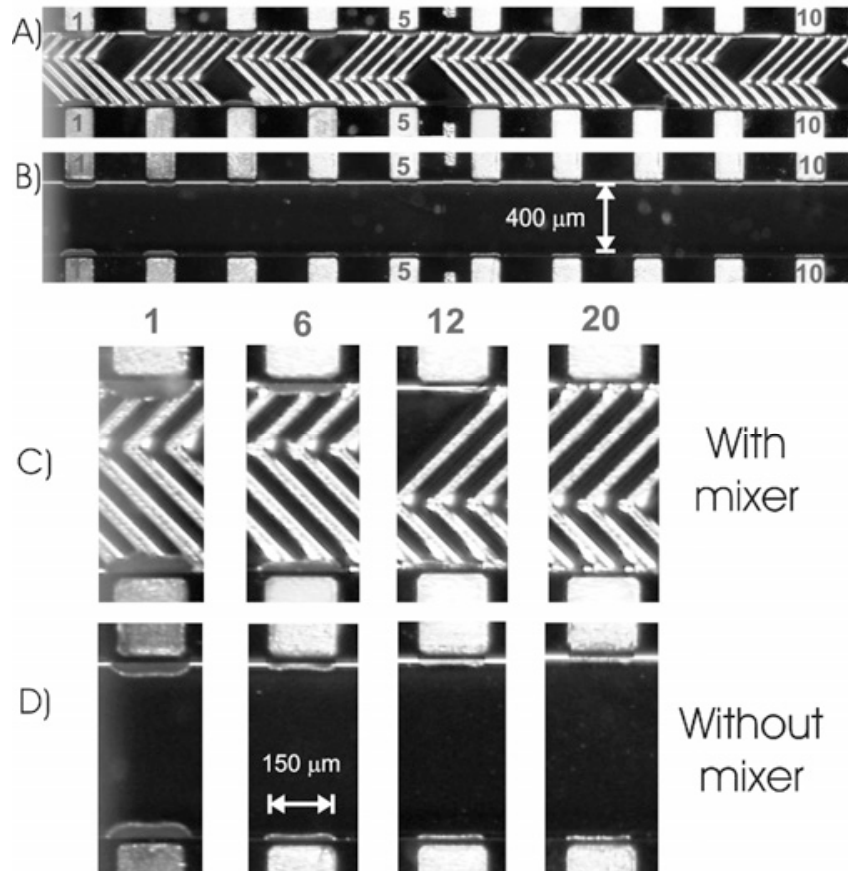

Figure 3: Micrographs taken after capture of beads in 400 $\mu \mathrm{m}$ wide channels with mixer structures A) and C) and without mixer structures $B$ ) and D). The micrographs in A) and $B$ ) show the first 10 elements (out of 30), and those in B) and $C$ ) are close-ups of elements 1, 6, 12, and 20. In the system with mixer, the amount of beads on the first element is high. It then gradually drops and after element 18 no captured beads are observed. In the system without mixer, the amount of beads on the first element is high. At the following elements it rapidly drops to an almost constant but nonzero level.

\section{RESULTS AND DISCUSSION}

\section{Qualitative observations}

Figure 3 shows micrographs of the beads captured during experiments for systems with and without mixer. Panels A) and $B$ ) of the figure show the first third of the channel for the two systems, while panels D) and E) show close-ups of selected magnetic elements after bead capture. A visual inspection of the system with mixer indicated that essentially all beads were captured within the first two thirds of the channel. For the system without mixer, beads were captured along the entire channel and many were even observed to escape the microsystem through the outlet during the experiment.

For both systems, essentially all beads were released when the external magnetic field was reduced to zero indicating close to zero magnetic remanence of the magnetic elements. During the magnetic bead release another advantage of the mixer structure became apparent. The mixer forces the beads from the sides of the channel to the central part of the channel, where the fluid flow velocity is higher, while they remain close to the sidewalls for the system without mixer. Hence, the beads were flushed out faster of the system with mixer. The micrographs in Figure 4 illustrate this effect. Moreover, as the beads are forced into the central flow the release is less sensitive to variations in the channel geometry (e.g., the increase in diameter at the outlet of the microsystem).

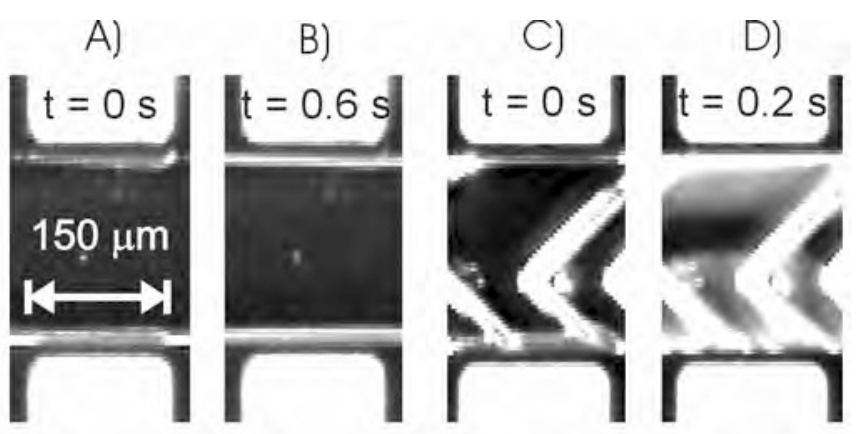

Figure 4. Micrographs of element 5 (out of 30) for bead release in systems with $200 \mu \mathrm{m}$ wide channels after the magnetic field is switched off at time $t=0 \mathrm{~s}$. Micrographs of a system without mixer at A) $t=0 \mathrm{~s}$ and $B$ ) $t=0.6 \mathrm{~s}$; the beads stay close to the sidewalls. Micrographs of a system with mixer at C) $t=0 \mathrm{~s}$ and D) $t=0.2 \mathrm{~s}$; the haze spreading from the magnetic elements in D) shows that the beads are forced away from the sidewalls.

\section{Bead quantification using magnetic measurements}

Figure 5 shows the measured saturation magnetic moments of bead solution volumes ranging from $V=0 \mu \mathrm{L}$ to $V=$ $200 \mu \mathrm{L}$. The inset shows a typical magnetic measurement performed on a sample with $V=100 \mu \mathrm{L}$ and illustrates the low noise of the measurements and the existence of a welldefined saturation magnetic moment. The measurements with zero volume were performed in several ways; some using an empty plastic tube, and others by adding pure water to a plastic tube and treating it like samples of bead solution. An experiment was also performed where pure water was run through the setup and collected. These measurements were performed to test that the handling of the samples was sufficiently clean and that no beads were present in the setup after cleaning between experiments. The average saturation magnetic moment of these in total 30 samples was $m=$ $0.1 \mathrm{nAm}^{2}$ (s.d. $=0.6 \mathrm{nAm}^{2}$ ). For $V=100 \mu \mathrm{L}$, which is close to that used in the capture experiments, the experiment was repeated 6 times and resulted in the average saturation magnetic moment of $m=59.4 \mathrm{nAm}^{2}$ (s.d. $=1.7 \mathrm{nAm}^{2}$ ). The increased standard deviation compared to $V=0$ results from the uncertainty in the bead concentration and/or the bead positioning in the VSM. The comparatively higher scatter of the data for $V=200 \mu \mathrm{L}$ in Figure 5 is very likely an effect of poor positioning of the beads, probably because they were not collected exactly at the bottom of the plastic tube. It is seen that the measured saturation magnetic moment is linearly correlated with $V$ with a negligible offset and hence that the method can be used to quantify the amount of beads collected in the plastic tubes. The absolute uncertainty on the bead saturation moment is estimated to $2 \mathrm{nAm}^{2}$. 


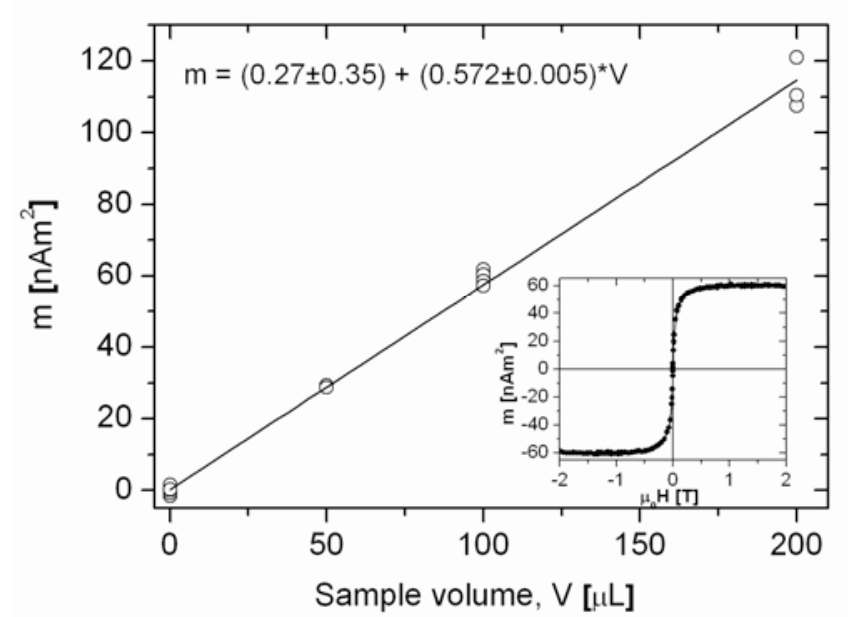

Figure 5: Measurements of the saturation magnetic moment for different volumes, $V$, of bead solution. It is seen that the magnetic moment is proportional to the sample volume. The inset shows the hysteresis loop measured for a sample with $V$ $=100 \mu \mathrm{L}$.

\section{Quantification of bead capture-and-release efficiency}

The three types of experiments described in the experimental section were carried out for the system with and without the mixer. The results are shown in Table 1.

\begin{tabular}{lcc} 
Result & With mixer & Without mixer \\
\hline $\begin{array}{l}\text { Bead collected at inlet } \\
m_{(1)}\left[\mathrm{nAm}^{2}\right]\end{array}$ & 40 & 40 \\
\hline $\begin{array}{l}\text { No bead capture } \\
m_{(2)}\left[\mathrm{nAm}^{2}\right]\end{array}$ & - & 40 \\
\hline $\begin{array}{l}\text { Bead capture-and-release } \\
m_{(3)}\left[\mathrm{nAm}^{2}\right]\end{array}$ & 36 & 23 \\
\hline$m_{(3)} / m_{(1)}$ & $90 \%$ & $58 \%$ \\
\hline $\begin{array}{l}\text { Table 1. Quantitative results } \\
\text { capture experiments. }\end{array}$ & &
\end{tabular}

From the table it is seen that $m_{(1)}$ corresponds to less than two thirds of the signal of $\approx 65 \mathrm{nAm}^{2}$ expected from the volume of the bead solution introduced into the fluidic set-up. This discrepancy is caused by beads that got stuck in the fluidic valves and tubes used for the bead solution and buffer flows. As these have large diameters compared to the microsystem, the flow velocity of the beads through these was so low that some of the beads settled due to gravitation. This was confirmed by sudden bead releases when air bubbles entered the fluidic set-up. We note that the performed experiments fully enable us to correct for the effect.

From Table 1, it is seen that $m_{(2)} \approx m_{(1)}$. This indicates that the amount of beads lost in the fluidic outlet is less than the experimental uncertainty. Hence, the bead capture-andrelease efficiency can be meaningfully defined as $m_{(3)} / m_{(1)}$.

The values of $m_{(3)}$ may be slightly lower than the saturation magnetic moment of the total amount of beads captured and released from the systems as only a limited fluid volume was collected in the experiments.
The quantitative data in Table 1 shows that the bead capture-and-release efficiency of the system with integrated mixer structures has greatly improved $(\sim 50 \%)$ compared to the system without the mixer structure.

\section{CONCLUSIONS}

Two different designs of passive magnetic bead separators were fabricated and characterized. The experiments showed that by inducing convection rolls in the integrated mixerseparator systems, the magnetic beads are moved into the capture zones and captured more efficiently. The mixer structure also results in a faster bead release by forcing the beads away from the slowly moving fluid near the channel sidewalls into the central part of the fluid flow. A new method for quantifying small amounts of beads based on magnetic measurements was proposed, validated and applied for measuring the capture-and-release efficiencies of the systems with and without mixer structures. The results indicate $\sim 50 \%$ increase in performance of the mixer system for the considered system geometry, beads and flow rate. In addition to the above mentioned advantages, the mixer structures also add extra functionality to the microfluidic system, as they can be used to, e.g., mix a flow of beads with the sample before the beads are captured.

\section{REFERENCES}

[1] M.A.M. Gijs, Microfluid Nanofluid, vol. 1, 22, 2004.

[2] A. Rida, and M. A. M. Gijs, Anal. Chem., vol. 76, 6238, 2004.

[3] R. Rong, J. W. Choi, and C. H. Ahn, In Proceedings IEEE Sixteenth Annual International Conference on Micro Electro Mechanical Systems, 530, 2003.

[4] K. Smistrup, T. Lund-Olesen, P. T. Tang, and M. F. Hansen, J. Appl. Phys., (In Press, 2006).

[5] J.-W. Choi, T. M. Liakopoulos, and C. H. Ahn, Biosens. Bioelectron., vol. 16, 416, 2001.

[6] K. Smistrup, O. Hansen, H. Bruus, and M. F. Hansen, J. Magn. Magn. Mater., vol. 293, 597, 2005.

[7] T. Deng, M. Prentiss, and G. M. Whitesides, Appl. Phys. Lett., vol. 80, 461, 2002.

[8] A. D. Stroock, S. K. W. Dertinger, A. Ajdari, I. Mezic, H. A. Stone, G. M. Whitesides, Science, vol. 295, 650, 2002.

[9] J. Wu, V. Quinn, and G. H. Bernstein, J. Micromech. Microeng., vol. 14, 576, 2004.

[10]Dynal Biotech, Norway.

[11]K. Smistrup, B. G. Kjeldsen, J. L. Reimers, M. Dufva, J. Petersen, and M. F. Hansen, Lab Chip, vol. 5, 1315, 2005. 